\title{
Bernanos e Montherlant: dai «Dialogues des Carmélites» a «Port-Royal»
}

\section{Pierangela Adinolfi}

\section{(2) OpenEdition}

12 Journals

\section{Edizione digitale}

URL: http://journals.openedition.org/studifrancesi/29841

DOI: 10.4000/studifrancesi.29841

ISSN: 2421-5856

\section{Editore}

Rosenberg \& Sellier

\section{Edizione cartacea}

Data di pubblicazione: 1 avril 2006

Paginazione: 43-55

ISSN: 0039-2944

\section{Notizia bibliografica digitale}

Pierangela Adinolfi, «Bernanos e Montherlant: dai «Dialogues des Carmélites» a «Port-Royal»», Studi Francesi [Online], 148 (XLX | I) | 2006, online dal 30 novembre 2015, consultato il 18 avril 2021. URL: http://journals.openedition.org/studifrancesi/29841; DOI: https://doi.org/10.4000/studifrancesi. 29841

\section{(c) (i) $\odot$}

Studi Francesi è distribuita con Licenza Creative Commons Attribuzione - Non commerciale - Non opere derivate 4.0 Internazionale. 


\section{Bernanos e Montherlant: dai «Dialogues des Carmélites» a «Port-Royal»}

Constatare la differenza delle idee che sono alla base del pensiero di due autori come Georges Bernanos e Henry de Montherlant potrebbe apparire un'operazione dall'esito facile e scontato, riassumibile con la semplice formula oppositiva secondo cui il credente Bernanos non ha nulla che lo avvicini all'ateo Montherlant. Un'analisi più approfondita rivela, al contrario, il terreno comune sul quale s'incontrano tematicamente i due scrittori francesi. Essi nutrono, infatti, la stessa profonda esigenza di riflettere sul senso dell'esistenza umana e sui grandi temi inerenti alla vita. Bernanos cattolico che scrive romanzi, e non romanziere cattolico come egli tiene a sottolineare, Montherlant autore, nella sua vasta e disparata produzione letteraria, di una trilogia cattolica, Le Maître de Santiago, La Ville dont le prince est un enfant, Port-Royal, percepiscono nel loro intimo l'urgente necessità del perfezionamento morale dell'essere umano e della ricerca dell'assoluto.

La vicinanza tematica che alimenta i nostri autori si rivela sorprendente in due opere teatrali e precisamente nei Dialogues des Carmélites di Georges Bernanos e in Port-Royal di Henry de Montherlant. Entrambe le pièces hanno come punto di partenza fatti storici documentati e circostanziati che possiedono come centro vitale il convento.

I Dialogues des Carmélites traggono la loro origine da una sceneggiatura cinematografica che Bernanos era stato incaricato di scrivere e quella stessa sceneggiatura a sua volta era stata ispirata alla celebre novella di Gertrud von Le Fort Die Lezte am Schafott (La Dernière à l'échafaud $)^{1}$. Nella novella della scrittrice tedesca è narrato il fatto storico delle sedici carmelitane di Compiègne che avendo fatto voto di martirio furono ghigliottinate in place de Grève il 17 luglio 1794. L'opera di Bernanos composta tra il 1947 ed il 1948 è, tuttavia, assai differente dal racconto tedesco. Lo scrittore francese, alla vigilia della propria morte, traspone nei Dialogues i temi che hanno caratterizzato i suoi romanzi. Il testo completo dei Dialogues venne ritrovato fra i manoscritti di Bernanos e pubblicato nel 1949 da Albert Béguin.

Il Port-Royal di Henry de Montherlant conosce diverse stesure: la prima tra il 1940 ed il 1942, la seconda, quella definitiva, nel 1953. La prima idea di scrivere una tragedia dedicata alle sofferenze delle suore gianseniste che nel 1664 furono perseguitate dalla Chiesa cattolica venne a Montherlant nel 1929, dopo la lettura del Port-Royal di Sainte-Beuve ed in particolare dopo aver letto la frase: «Port-Royal ne fut qu'un retour et un redoublement de foi à la divinité de Jésus-Christ» ${ }^{2}$. Nella composizione della sua pièce, Montherlant tiene in considerazione i documenti storici relativi ai fatti e le lettere scritte dalle suore stesse, contenute nella Relation di quanto accadde nei giorni della persecuzione ${ }^{3}$.

Questi i punti di partenza di due opere teatrali a carattere religioso che pur essendo diverse nella struttura, i Dialogues in cinque atti e Port-Royal in un atto unico, sono accomunate da un grande tema: la paura.

(1) Sulla genesi dei Dialogues des Carmélites, cfr. Notes et variantes, in G. Bernanos, Euvres romanesques suivies de Dialogues des Carmélites, Préface par G. PICON, texte et variantes établis par A. BÉGuin, notes par M. Estève, Paris, Gallimard, [1961] 2002 («Bibliothèque de la Pléiade»), pp. 1918-1921.
(2) Cfr. H. De Montherlant, Du côté de la souffrance, in Théâtre, Préface de J. DE LAPRADE, préface complémentaire de PH. DE SAINT RoBERT, Paris, Gallimard, [1971] 1995 («Bibliothèque de la Pléiade»), p. 917.

(3) Cfr. Ibid., note 1, 2, pp. 918-919. 
Bernanos indica la paura quale tema dominante la sua pièce già a partire dall'epigrafe che egli trae dalla Joie: «En un sens, [...] la Peur est tout de même la fille de Dieu, rachetée la nuit du Vendredi-Saint. Elle n'est pas belle à voir - non! - tantôt raillée, tantôt maudite, renoncée par tous... Et cependant, ne vous y trompez pas: elle est au chevet de chaque agonie, elle intercède pour l'homme» ${ }^{4}$. In questo contesto e in quello dei Dialogues, la paura, anch'essa «fille de Dieu», svolge una funzione fondamentale e cioè diventa strumento di salvezza spirituale nel momento dell'agonia umana. Elemento di congiuntura, insieme con la sofferenza, della natura umana e dell'essenza divina, la paura offre all'uomo la possibilità della santificazione e della vita eterna. Componente della fragilità e della debolezza umane, provate da Gesù Cristo nell'Orto degli Ulivi e durante la Passione, la paura è ciò che consente all'individuo di partecipare alla Santa Agonia e di purificare e fortificare la propria anima per accedere alla vita ultraterrena ed alla gioia della grazia divina.

Eroina protagonista dei Dialogues, pervasa da una paura quasi patologica, è Blanche de la Force. La natura fragile e paurosa di Blanche è descritta con cura sin dall'inizio dei Dialogues: «L'humeur de Blanche a quelque chose qui passe l'entendement ordinaire»5. Anche qui Bernanos, come negli altri suoi romanzi, offre la rappresentazione psico-fisica della paura che acquisisce intensità e connotazioni differenti, a seconda che sia legata al peccato o alla grazia, sino a trasformarsi nell'angoscia metafisica. Blanche, insieme con l'abbé Donissan, l'abbé Chevance, Chantal de Clergerie, il curé d'Ambricourt, rientra nella schiera dei personaggi bernanosiani colti dalla timidezza e dal timore che diventano nei diversi contesti paura, angoscia e terrore. Il temperamento malinconico e pauroso di Blanche: «Je meurs chaque nuit pour ressusciter chaque matin» ${ }^{6}$ è subito posto in relazione con l'Agonia di Gesù: «C'est qu'il n'y a jamais eu qu'un seul matin [...] celui de Pâques. Mais chaque nuit où l'on entre est celle de la Très Sainte Agonie»' ${ }^{7}$. Nell'evoluzione spirituale di Blanche, come in quella di tutti i personaggi santi bernanosiani, è insita la condivisione dello stato di angoscia della Santa Agonia del Cristo. Il sentimento della paura è pertanto strettamente connesso con l'idea della morte e della solitudine del Dio che si è fatto uomo: «Nul être vivant n'entra dans la mort aussi seul et aussi désarmé» ${ }^{8}$. Nell'angoscia sublime di Dio percepita nell'Orto degli Ulivi, la paura ed il coraggio non hanno più distinzione: «Lorsqu'on les considère de ce jardin de Gethsémani où fut divinisée, en le Cœur Adorable du Seigneur, toute l'angoisse humaine, la distinction entre la peur et le courage ne me paraît pas loin d'être superflue et ils nous apparaissent l'un et l'autre comme des colifichets de luxe»' . Ciò che conta è il compimento della volontà del Padre:

Le courage peut bien être aussi une fantasmagorie du démon. Une autre. Chacun de nous risque ainsi de se débattre avec son courage ou sa peur comme un fou qui joue avec son ombre. Une seule chose importe, c'est que, braves ou lâches, nous nous trouvions toujours là où Dieu nous veut, nous fiant à lui pour le reste. Oui, il n'est d'autre remède à la peur que de se jeter à corps perdu dans la volonté de Dieu, ainsi qu'un cerf poursuivi par les chiens, dans l'eau fraîche et noire ${ }^{10}$.

In questo contesto, la paura umana, la paura di Blanche, è considerata uno strumento nelle mani di Dio, utile ad aumentare l'intensità della prova e del sacrificio:

(4) Cfr. G. Bernanos, La Joie, in Euvres romanesques, cit., p. 675 .

(5) G. Bernanos, Dialogues des Carmélites, in Euvres romanesques, cit., p. 1570.

(6) Ibid., p. 1575.
(7) Ibid.

(8) Ibid., p. 1668.

(9) Ibid., p. 1653.

(10) Ibid., p. 1661-1662. 
«La fragilité de ma nature n'est pas une simple humiliation qu'il m'impose, mais le signe de sa volontà sur sa paure servante» ${ }^{11}$. La debolezza si tramuta in forza, la volontà umana aderisce alla volontà divina e l'individuo riesce, infine, a tuffarsi nell'«eau fraîche et noire», simbolo della fede consolidata dispensatrice di pace e di serenità. Data la fragilità della sua natura, Blanche si rifugia nel Carmelo perché questo le sembra l'unico luogo in cui poter vivere. Anche qui, tuttavia, conosce la violenza del mondo che incombe sul convento a causa della Rivoluzione. In questo contesto, Dio vuol provare non la forza, ma la debolezza di Blanche che nella piena accettazione della volontà divina e nel sacrificio spontaneo di sé troverà la chiave che apre le porte della salvezza eterna. Il dubbio è sconfitto: «Où je suis, rien ne peut m'atteindre» ${ }^{12}$ e la finale serenità di Blanche è la testimonianza dell'alto valore metafisico acquisito dalla morte nella visione bernanosiana. La paura della morte è legittima ed è parte inalienabile dell'essere umano. Bernanos offre un esempio emblematico della difficoltà del trapasso attraverso la descrizione della morte della Priora che sembra conoscere soltanto l'angoscia della Santa Agonia e non la gioia della grazia che ne deriva: «L'angoisse adhère à ma peau comme un masque de cire... Oh! que ne puis-je arracher ce masque avec mes ongles! $\gg^{13}$. Tuttavia, nonostante l'alto senso tragico della morte individuale in cui l'essere umano si trova solo con la propria paura, Bernanos pone in quest'evento la speranza e la luce finali, segni della fede in Dio e nell'immortalità dell'anima. La pace, la libertà e la vera gioia si acquisiscono attraverso la morte e l'accettazione dell'angoscia dell'agonia. Rappresentate attraverso caratteristiche psicologiche individuali, volte a mettere in luce le differenze e le paure specifiche di ciascuna, le suore del Carmelo aderiscono tutte alla Passione del Cristo attraverso il voto del martirio pronunciato per meritare il mantenimento del Carmelo e la salvezza della Patria. Le martiri del Carmelo vanno incontro alla morte per acquisire il dono della grazia non per sé, ma per altri, per la Francia e per la comunità religiosa. È possibile quindi morire per gli altri, e nella prospettiva della Comunione dei Santi, la morte serena di Blanche riscatta il difficile e angoscioso trapasso della Priora. Le anime eccezionali di Compiègne salgono al patibolo intonando il Salve Regina ed il Veni Creator. In questa rappresentazione finale, Bernanos sopprime il dialogo per lasciare unicamente la descrizione di quanto accade sulla scena. La pace della libertà acquisita nella fede in Dio e nell'accettazione dell'angoscia è percepibile attraverso il canto delle suore che una ad una, come agnelli sacrificali, passano sotto la lama della ghigliottina. La voce del coro si fa sempre più flebile finché non ne rimane che una: la voce di Blanche, più ferma e più risoluta delle altre, simbolo dell'innocenza e della purezza del fanciullo che sapendo di essere accolto dall'abbraccio del Padre non conosce la paura: «Mais à cet instant, partant d'un autre coin de la grande place, une nouvelle voix s'élève, plus nette, plus résolue encore que les autres, avec pourtant quelque chose d'enfantin. Et on voit s'avancer vers l'échafaud, à travers la foule qui s'écarte, interdite, la petite Blanche de la Force. Son visage semble dépouillé de toute crainte» ${ }^{14}$.

In tutta la pièce, Bernanos compie costantemente la propria riflessione sulla morte che, come abbiamo potuto constatare, è saldamente connessa con l'idea della paura. L'autore esprime la legittimità della paura della morte quale evento intimo e personale in cui l'essere umano, come alla nascita, si trova solo con se stesso. Ognuno muore come può, da santo, da eroe, da martire o in preda all'angoscia. Agli occhi di Dio ciò che conta non è la morte valorosa, poiché in essa è racchiusa la vanità umana, bensì la purezza dell'anima che aderisce alla volontà del Padre nella spontanea ac- 
cettazione dell'angoscia dell'agonia. In prossimità della morte, le anime vicine a Dio ricuperano l'innocenza dell'infanzia persa nell'età adulta e faticosamente ricercata per tutta la vita:

Cette simplicité de l'âme, ce tendre abandon à la Majesté divine [...] nous consacrons notre vie à l'acquérir, ou à le retrouver si nous l'avons connu, car c'est un don de l'enfance qui le plus souvent ne survit pas à l'enfance... Une fois sorti de l'enfance, il faut très longtemps souffrir pour y rentrer, comme tout au bout de la nuit, on retrouve une autre aurore. Suis-je [la Prieure] redevenue enfant ? $^{15}$.

Nell'estremo evento sublime e terribile è quindi riposta, per Bernanos, la possibilità del riscatto umano e la convinzione dell'esistenza della vita eterna.

In maniera differente, benché contraddistinti dallo stesso senso del mistero che li avvolge, sono i concetti di paura e di morte nella visione montherlantiana. In PortRoyal, Montherlant traccia il doppio percorso ascendente e discendente di due anime verso la Luce e verso le Tenebre: «La Sœur Françoise est mise, à l'improviste, devant la "lumière". La Sœur Angélique s'achemine, d'un cours logique et prévu, vers "les Portes des Ténèbres" $\gg{ }^{16}$. La causa scatenante che induce le due anime conventuali a compiere il tragitto spirituale opposto è la richiesta della firma del Formulario redatto dalla Chiesa cattolica in cui vengono condannate cinque affermazioni di Giansenio ritenute eretiche. In questo contesto, la figura femminile che subito risalta in mezzo alle altre del monastero di Port-Royal è quella di Sœur Angélique. Montherlant focalizza il suo interesse sulla crisi di dubbio religioso che turba profondamente la suora fino a destabilizzare le fondamenta stesse della sua fede. Lo sconvolgimento provocato dal dubbio induce il terrore del demonio. La paura nata dall'incertezza della fede determina l'inarrestabile caduta verso un abisso di tenebre che lascia palesare, quale tragico esito della vicenda, la morte al di fuori del monastero. Secondo il suo stile, Montherlant descrive lucidamente il travaglio interiore di un'anima lacerata dal dissidio tra ciò che l'abito impone e ciò che la coscienza avverte. L'inquietudine e la paura diventano palpabili in tutta la pièce ma non consentono, nel caso di Sœur Angélique, di approdare alla salvezza eterna. Come spesso accade nel teatro montherlantiano, Henry rappresenta un personaggio tragico che inevitabilmente soccombe schiacciato dalla forza dirompente degli eventi. È motivo di interesse notare qui l'attuazione dello schema "crisi di dubbio religioso - paura - caduta verso le tenebre". Tale dinamica è, infatti, ampiamente rintracciabile nell'opera romanzesca bernanosiana, ad esempio in Sous le Soleil de Satan e nel Journal d'un curé de campagne, in cui il dubbio e la paura, effetti della tentazione diabolica, sconvolgono i personaggi santi come quelli mediocri, basti pensare all'abbé Donissan, a Mouchette ed al curé d'Ambricourt.

Il caso di un'altra figura bernanosiana, l'abbé Cénabre nell'Imposture, è quanto mai vicino a quello di Sœur Angélique, poiché questo prete mediocre conosce il dubbio, giunge alla constatazione dell'assenza di fede, scivola nel gorgo del terrore ed approda alla follia. Se i personaggi descritti da Bernanos compiono un percorso spirituale analogo a quello tracciato da Montherlant in Port-Royal a proposito di Sœur Angélique sulla strada del precipizio, ad essi appartiene, tuttavia, la doppia possibilità della salvezza o della dannazione: elevarsi verso la luce eterna o inabissarsi nell'oscurità delle tenebre. L'ascesi di Sœur Françoise è, in realtà, un cammino quasi privo di paura che consente il raggiungimento della pace in maniera più facile ed immediata. La giovane suora non è sconvolta dal dubbio e lascia subito trasparire il 
consolidamento della propria fede. La sua innocenza infantile la accomuna a Sœur Constance dei Dialogues des Carmélites: entrambe appaiono, sin dall'inizio, come il simbolo della purezza e della fede proprie dell'esprit d'enfance. I maggiori ostacoli ed i più grandi turbamenti sono, pertanto, destinati dagli autori a Blanche de la Force ed a Sœur Angélique, le quali andranno incontro alla morte dopo essere approdate a convinzioni spirituali opposte.

Il concetto della morte è espresso da Bernanos in maniera più esplicita e vibrante: egli "accompagna" le suore ad una ad una sulle scale del patibolo. Montherlant, invece, si limita all'evocazione finale della morte attraverso la rappresentazione delle suore raccolte in preghiera mentre odono il suono dell' «heure none», l'ora della morte di Cristo. Il tema della morte è profondamente sentito da entrambi gli autori ed il contesto di queste due rappresentazioni religiose, Dialogues des Carmélites e Port-Royal, offre agli scrittori la possibilità di esprimere la propria personale concezione della morte. Bernanos, come abbiamo già detto, attribuisce all'agonia umana un alto significato metafisico associandola all'Agonia del Cristo e dando valore alla paura presente al capezzale di ogni uomo. L'accettazione di tale angoscia consente il raggiungimento della pace e l'accesso al Regno dei Cieli, come accade per Blanche de la Force. Montherlant, al contrario, pur considerando la morte un evento capitale nell'esistenza dell'essere umano, nella sua pièce sottolinea l'importanza e la difficoltà del controllo della paura della morte: se per Bernanos è fondamentale l'accettazione di quella paura quale testimonianza dell'adesione umana alla volontà del Padre, per Montherlant, il cui pensiero è fortemente imbevuto di stoicismo, è altrettanto fondamentale la maîtrise degli eventi, inclusa la propria morte, ad opera dell'individuo. Henry espone ampiamente nell'opera narrativa, saggistica e nei Carnets la sua riflessione sulla morte e sul suicidio, concetti per lui solidamente connessi alla dignità umana. Egli stesso diventa testimone del suo pensiero togliendosi la vita nel momento in cui questa perde per lui le irrinunciabili caratteristiche di autonomia e dignità. Di fronte all'agonia umana, Montherlant percepisce la vacuità dell'esistenza, se non condotta all'insegna del perseguimento del proprio plaisir e del proprio bonbeur, ed il Nulla che segue la morte. La vita per lui non è altro che «une récréation entre deux néants $\gg^{17}$. Bernanos, invece, ne esalta l'aspetto sacro e sublime. Entrambi sono rapiti dal grande mistero dell'assoluto, l'uno senza mai penetrarvi fino in fondo, l'altro tuffandosi «à corps perdu dans la volonté de Dieu [...] dans l'eau fraîche et noire». Nel contesto di Port-Royal, il prevalere del dubbio in Sœur Angélique determina la messa in luce dell'aspetto più razionale dell'essere umano. Attraverso la centralità di questo personaggio, Montherlant evidenzia anche la funzione negativa che la paura e la sofferenza rivestono per lui. Come Blanche de la Force anche Sœur Angélique può dire: «J'ai peur de tout» ${ }^{18}$, ma la sua paura è causata dal dubbio religioso. Chi infatti ha veramente fede possiede un animo quieto e sereno come sostiene Mère Agnès: «Tout ce que nous faisons de bon est fait avec un esprit en paix. L'inquiétude est un grand témoignage du peu de profit que nous faisons de la sainte Communion, et quiconque ne s'estime heureux en ce monde ne le peut être dans l'autre. Vous croyez en Dieu, et vous craignez quelque chose? $\gg^{19}$. Ancora una volta si trovano qui elementi comuni con tutta l'opera bernanosiana: il bonbeur dei santi e di quanti possiedono l'esprit d'enfance, si pensi alle parole e all'atteggiamento del curé de Torcy nel Journal d'un curé de campagne, prove della fede salda e matura che infonde la pace nel

(17) Su questo tema rimandiamo al nostro studio «Sur mes derniers Carnets»: un testo inedito di Henry de Montherlant, in «Studi francesi», n. 132, settembre-dicembre 2000, pp. 527-533; e anche a H. DE MonTHerlant, «Une récréation entre deux néants»: lettere inedite a Luigi Bàccolo, a cura di P. Adinolfi, Torino, Thélème, 2002, pp. 107-110.

(18) H. De Montherlant, Port-Royal, in Théatre, cit., p. 877.

(19) Ibid. 
cuore dei puri. L'altro elemento comune con l'opera di Bernanos rintracciabile nel Port-Royal di Montherlant è la messa in discussione di se stessi, della propria natura, delle proprie capacità, della forza che il personaggio ritiene sempre di non possedere a sufficienza per superare gli ostacoli presenti sul proprio cammino. Sœur Angélique risponde alle parole di Mère Agnès poco prima citate: «Je me crains. - Je crains aussi tout le reste $\gg^{20}$. Sœur Angélique teme l'infeconda paura, concepita come sofferenza dell'anima, che può condurla alla perdizione:

Il y a une souffrance qui n'est pas féconde, une souffrance morte, et qui entraîne dans sa mort tout ce qu'elle trouve en l'âme autour d'elle. Vous [Mère Agnès] parlez des vérités de l'Église. Mais s'il y avait une souffrance qui allât jusqu'à vous obscurcir ces vérités? Je connais de nos sœurs qu'un certain excès de peines met dans un état si étrange qu'il leur semble alors qu'elles ne croient plus en Dieu ${ }^{21}$.

In queste parole, Sœur Angélique allude alla propria esperienza di dolore, derivato dalla realtà della persecuzione religiosa e dal dubbio della fede, che la spingerà fino alle «Portes des Ténèbres». Al contrario di quanto accade in Bernanos, per il quale la sofferenza possiede l'alto valore cristiano della purificazione e della santificazione, se non ostentata e fine a se stessa altrimenti diverrebbe un peccato di orgoglio, Montherlant rappresenta qui l'aspetto più arido e sterile dell'intensa sofferenza umana che nel caso di Sœur Angélique ha come solo esito la perdita della fede e della salvezza eterna. Rilevante è il fatto che Montherlant faccia pronunciare le parole di lode nei confronti della sofferenza cristiana dall'Arcivescovo di Parigi, simbolo del pericolo e del Male esterno che minaccia la sicurezza e la salvezza spirituale del monastero come il potere rivoluzionario minaccia il Carmelo nei Dialogues. Rivolgendosi a Sœur Angélique, il prelato afferma: «On a beau souffrir, si on est hors de l'Église, cela ne sert de rien. Combien y a-t-il eu d'hérétiques qui se sont exposés au supplice, que dis-je? qui le convoitaient, et qui maintenant brûlent dans le feu? Le diable lui aussi a ses martyrs ${ }^{22}$. Colui che considera la sofferenza delle martiri di Port-Royal una sofferenza diabolica è proprio il personaggio nel quale Montherlant concentra la rappresentazione simbolica della tentazione demoniaca.

Stabilendo un altro forte punto di contatto con l'opera e la pièce bernanosiane, nella suggestiva descrizione del Male incombente, Montherlant utilizza una vasta simbologia facilmente rintracciabile nello stile visionario bernanosiano. Entrambi gli autori introducono, infatti, la presenza oscura del Maligno a partire dalla rappresentazione dell'orage che si teme si abbatta tanto sul Carmelo di Compiègne quanto sul monastero di Port-Royal.

Nei Dialogues des Carmélites come nel resto della sua opera, Bernanos ricorre frequentemente alla simbologia dell'acqua per conferire all'elemento naturale sia il significato positivo della purezza, della gioia e della fede in Dio sia quello negativo del peccato, della degradazione dell'anima e della contaminazione del Male. L'acqua fangosa e densa oppure la fitta pioggia che ricopre la natura ed i villaggi possiedono questa connotazione. Nei Dialogues più volte è annunciata ed attesa una tempesta dal carico di nubi nere, che nulla hanno a che fare con «l'eau fraîche et noire» della volontà divina. Qui l'orage simboleggia il Male cioè la distruzione delle istituzioni religiose ad opera della Rivoluzione francese. Sui personaggi aleggia la nera minaccia della tempesta: «Je [le marquis] dirais volontiers qu'un orage menace, si nous n'étions si tôt dans la saison. Le ciel s'est couvert brusquement, tandis que vous 
[Blanche] parliez» ${ }^{23}$. La Priora, prima di morire si sente oppressa dal caldo della stagione ed allude all'orage quale evento inevitabile che porterà con sé la tragedia finale, ma anche la liberazione spirituale delle suore: «Les premières chaleurs m'ont toujours fait grand mal [...]. Il faut que cet orage crève, et vous [le médecin] me verrez déjà bien soulagée...» ${ }^{24}$. Il presagio di morte associato al simbolico orage che sta per abbattersi sul convento si chiarisce nella profezia della Priora che sta morendo: «Je viens de voir notre chapelle vide et profanée - oh! oh! - l'autel fendu en deux, les vases sacrés jonchant le sol, de la paille et du sang sur les dalles... Oh! oh! Dieu nous délaisse! Dieu nous renonce! $\gg^{25}$.

Anche Montherlant, in Port-Royal, fa uso dell'orage per annunciare il pericolo in arrivo. Tuttavia, benché egli evochi l'orage attraverso i racconti ed i sogni premonitori delle suore, la tempesta è soppiantata da un altro temibile evento naturale che ne acquisisce il sinistro significato: la siccità. L'aridità, il caldo soffocante, la luce accecante del mese di agosto rimandano alle condizioni climatiche in cui, nella Joie, Bernanos fa agire i suoi personaggi ed in particolar modo Chantal de Clergerie e la nera figura della nonna, nell'abbagliante luce e nella calura opprimente della canicola estiva: soltanto nella Joie, attraverso la luce arriva la tentazione demoniaca. In tutte le altre opere bernanosiane, il demonio arriva nell'oscurità. Nella pièce montherlantiana, l'aridità e la «sécheresse» sono veicoli del male:

LA Sceur Louise: Chaque fois que nous avons reçu une visite de la police, il y a eu un orage dans les deux jours qui ont précédé. Les nuées vont de Paris à Rome, et de Rome à Paris, mais c'est toujours sur Paris qu'elles éclatent. Aujourd'hui, ciel pur. Et quel soleil!

La Sceur Gabrielle: Vous souvenez-vous, ma Sœur? C'est le mercredi saint de 1661 que se tint ce Conseil du Roi qui fut le commencement ou plutôt la reprise de nos malheurs. Or, la nuit suivante, sans en rien savoir, sans connaître en nulle façon que nous étions menacées, ma Sœur Marie-Claire vit en songe une nuée très obscure, et dans cette nuée une bête épouvantable, d'une noirceur extraordinaire, qui prit sa course vers notre monastère, avec d'horribles rugissements. Après avoir rugi sur notre monastère, le monstre se remit à courir de toute sa force vers le Louvre, où il redoubla ses rugissements. Et de là reprit sa course tout en fureur vers le monastère, et notre sœur se réveilla transie d'effroi. L'histoire de Port-Royal est un épisode de la lutte très ancienne de l'Esprit-Saint contre la Bête.

La S(eur Angélique: Eh bien! Quelqu'une d'entre vous a-t-elle eu ces jours-ci de semblables songes? (Les sæurs font non de la tête.) Ni orages, ni songes de nuées. Les hommes sont donc tranquilles. Mais s'il y avait des orages et des nuées, ils sont aussi de la pluie. Et la pluie défait la sécheresse. Et c'est la sécheresse qui est notre pire ennemie. Tout plutôt que la sécheresse. N'oubliez jamais cela ${ }^{26}$.

Come nella Joie bernanosiana, anche qui la luminosità intensa della luce d'agosto e l'oscurità e la «noirceur» della «bête» peccaminosa sono inizialmente entrambe presenti. Montherlant fa un esplicito riferimento alla lotta tra il Bene ed il Male. La carica simbolica dell'orage e delle nuées viene in seguito gradatamente trasferita nella torrida calura del mese di agosto e nel disagio spirituale percepito da Sœur Angélique. La monaca ricerca la pace e la sicurezza della penombra chiudendo le imposte e attendendo il calar del sole. Nell'ora centrale e più calda della giornata è anche previsto, come vedremo, l'arrivo dei «démons du milieu du jour», i demoni tentatori che esigono la firma del Formulario. La minaccia ed il pericolo si palesano con l'intensificarsi dei raggi del sole, la calma e la serenità ritornano col fresco e col buio:

(23) G. Bernanos, Dialogues des Carmélites, in Euvres romanesques, cit., p. 1574.

(24) Ibid., p. 1589.
(25) Ibid., p. 1604.

(26) H. DE Montherlant, Port-Royal, in Théâtre, cit., pp. 863-864. 
La Sceur Angélique: Aujourd'hui, le 26 d'août... Le milieu de l'année, et le milieu du jour. L'heure des démons du milieu du jour. Je ne sais ce qui est le pis: ou le milieu du jour, ou le réveil, avec la journée qu'il va falloir charger. Ce grand silence d'août. Il y a un silence et un abandon, en août, qui me figurent terriblement le silence et l'abandon de Dieu. Quand le fort et le chaud du jour seront passés, vers cinq heures, je serai mieux. Et puis, à cinq heures, on est tranquille jusqu'au lendemain matin: il ne se passe rien la nuit. La menace monte avec le soleil ${ }^{27}$.

Sœur Angélique sente l'abbandono di Dio ed è colpita da una febbre d'ansia che la consuma lentamente. La monaca nutre in sé un grande desiderio di settembre: «Comme je l'attends, ce premier de septembre! Encore cinq jours! Alors, au bout de nos jardins, on recommencera de sentir l'odeur des champs. Septembre se relâche. Mais août est tout dur et en feu $\gg^{28}$. Se settembre rappresenta, pertanto, il mese della distensione e della frescura, momento in cui si allontanano le forze del male, agosto, come abbiamo già detto, è il mese demoniaco, «tout dur et en feu». Al contrario di quanto accade nella simbologia bernanosiana dove il fuoco, ampiamente utilizzato, generalmente costituisce l'elemento purificatore di Dio, qui il caldo e la vivida fiamma del fuoco rappresentano entrambi le forze del male. Emblematico è il sogno premonitore di Sœur Angélique in cui la monaca presagisce lo smarrimento causato dal proprio dubbio. In questo sogno l'oscurità della notte è affiancata a quella delle nubi tempestose, al contempo nere ed in fiamme:

Je tâchais d'atteindre Port-Royal-des-Champs, seule, à pied, de nuit, sans voir les chemins. J'y arrivais, j'entrais par une fenêtre du rez-de-chaussée, et je trouvais plusieurs de nos sœurs de Paris, notamment Sœur Sinclétique, très tristes. Je me retournais vers une fenêtre, et je voyais l'air tout en feu, et une nuée à la fois noire et enflammée, comme on en voit dans ces affreux mois de l'été, quand il va faire un orage. La Sœur Sinclétique, regardant cela, dit, avec un grand soupir: «Ah! quelle nuit nous aurons encore!» Je ne savais ce qu'elle voulait dire, mais elle me fit peur. Et, étant sortie, parce que je voulais aller adorer le Saint-Sacrement, il faisait de nouveau si nuit que je ne reconnaissais plus les chemins et je me réveillai sans achever mon songe $e^{29}$.

Le tenebre notturne e il disorientamento di Sœur Angélique, la sua incapacità di riconoscere «les chemins» data la profonda oscurità della notte, sono la simbolica rappresentazione dell'impossibilità della suora di scegliere la strada della fede: in lei si è già insinuato il dubbio e con il dubbio la paura. Ancora una volta, qui come in altri luoghi della pièce, Montherlant conferisce sia all'oscurità che alla luce, al cielo nero ed in fiamme della tempesta, come alla notte tentatrice, il potere demoniaco, lo stesso potere che appartiene ai «démons du milieu du jour» che fanno trionfanti il loro ingresso in mezzo ai raggi del sole:

On entend des bruits divers dans la cour, bruit de voix, ensuite le roulement d'un carrosse avec des sabots et des grelots de chevaux. La Soeur Angélique dit, dans un souffle: «Le char de feu...» La Mère Agnès, qui est proche de la fenêtre, se lève, regarde, et pousse un cri: «M. L'Archevêque!»

La Seeur Angélique, d'une voix blanche: Ma Mère, vous voyez l'horloge, dans la cour. Quelle heure marque-t-elle?

LA MÈRE AgNÈs: Midi.

$[\ldots]$ 
Les douze coups de midi sonnent à l'horloge.

[...]

Les cinq ecclésiastiques s'arrêtent sur le seuil de la pièce; ils sont pris dans le rayon de soleil venant de la fenêtre comme dans le feu d'un projecteur. Avec leurs ors, leurs rouges, leurs noirs, ils semblent une assemblée de magnifiques et un peu monstreux insects ${ }^{30}$.

Gli ecclesiastici che giungono nella luce del sole sono scenograficamente rappresentati in maniera suggestiva: i loro colori, oro, rosso, nero, rimandano, ancora una volta, alla luce tentatrice, alle fiamme dell'Inferno ed all'oscurità della notte veicolo del male. È noto quanta parte abbia la notte nella simbologia bernanosiana del male, basti pensare all'episodio dell'incontro dell'abbé Donissan col maquignon in Sous le Soleil de Satan. Nel Soleil, l'abbé Donissan, con l'intensificarsi delle tenebre, perde il cammino ed inconsapevolmente si dirige verso il diavolo tentatore. Lo stesso titolo del romanzo, Sous le Soleil de Satan, riunisce quasi ossimoricamente elementi opposti: il sole, e quindi la luce, e Satana, Signore del Male e delle Tenebre.

Nel contesto di Port-Royal, Montherlant utilizza ancora una volta la simbologia del "chiaro" e dello "scuro" e precisamente nella scena finale della pièce, nel momento in cui appaiono le Scurs de la nuit e cioè le Sours de la Visitation Sainte-Marie, giunte al monastero in sostituzione delle suore gianseniste espulse, per non aver firmato il Formulario, fra cui è inclusa anche Sœur Angélique. Se le monache di PortRoyal sono vestite di bianco, le Sours de la nuit sono vestite di nero ed il loro arrivo al monastero precede di poco il calar della notte ${ }^{31}$.

In questo contesto di luci ed ombre si svolge la tragedia montherlantiana. Se da un lato Montherlant rappresenta l'aspetto più scettico e razionale del dubbio, dall'altro lato mette in scena l'incapacità umana di controllare le proprie emozioni e le proprie passioni. Come accade nella tragedia greca, gli eroi di Montherlant, si pensi a Pasiphaé, non sono in grado, benché esercitino la propria volontà che spesso coincide con la passione nefasta, di mâ̂triser il côté più irrazionale specifico della natura umana ed affrontano tutte le conseguenze delle proprie azioni.

Il caso di Sœur Angélique è particolarmente tragico poiché, pur non essendo sostenuta dalla pace e dalla forza della fede, va incontro alla morte per non aver firmato il Formulario. Montherlant rappresenta in maniera emblematica il dramma che si consuma all'interno di Sœur Angélique dal momento che intende esprimere la mescolanza di bene e di male plausibilmente presente in una stessa anima: «Il y a de tout en certaines âmes. Et parfois dans le même moment» $\aleph^{32}$. Attraverso l'intima lacerazione di Sœur Angélique, quel dissidio che la conduce alla «tentation du désespoir» ${ }^{33}$, è possibile cogliere una nuova analogia coi personaggi bernanosiani. Anche Bernanos, infatti, crea dei personaggi in cui la lotta fra il bene ed il male e il dolore che ne deriva sono sempre presenti. In essi il dubbio latente va combattuto e la tentazione della disperazione e del suicidio li visita costantemente. Come Sœur Angélique esclude il coraggio e la speranza della grazia e mette in estremo pericolo la sua fede, arrivando al punto di non riuscire più a pregare, così l'abbé Donissan conosce il dubbio ed una parte del Soleil è intitolata «La tentation du

(30) Ibid., pp. 886-887.

(31) Cfr. Ibid., pp. 915-916.

(32) Ibid., p. 913.

(33) Montherlant esprime l'intensa disperazione di Sœur Angélique in maniera allusiva, non descrivendo il limite estremo al quale la suora potrebbe arrivare. La disperazione e il dubbio di Angélique si manifestano anche attraverso l'incapacità di pregare: «Quand les paroles de L'Écriture, qui tant de fois vous ont donné tant de force, ne vous donneront plus rien; quand, tenant cela dans ses doigts (elle tient le chapelet de sa ceinture), on n'aura plus envie de le porter à sa bouche; quand il vous viendra des idées si effroyables que vous y apprendrez ce que c'est que le désespoir, et par où l'on y va, et quelle est la tentation qui peut naître de ce désespoir... [...] Prier? Prier Dieu? Mais si Dieu...? Je me perds quand j'y pense» (Port-Royal, cit., pp. 884-885). 
désespoir», la contessa, nel Journal, pecca contro la speranza dopo la morte del figlioletto, il curé d'Ambricourt sente la notte penetrare all'interno della propria anima ed ogni tentativo di pregare risulta per lui inutile:

Encore une nuit affreuse, un sommeil coupé de cauchemars. [...] Jamais je ne me suis tant efforcé de prier, d'abord posément, calmement, puis avec une sorte de violence concentrée, farouche, et enfin - le sang-froid retrouvé à grand-peine - avec une volonté presque désespérée (ce dernier mot me fait horreur), un emportement de volonté, dont tout mon cœur tremblait d'angoisse. Rien. [...] J'aspire la nuit, la nuit entre en moi par je ne sais quelle inconcevable, quelle inimaginable brèche de l'âme. Je suis moi-même nuit ${ }^{34}$.

Sempre nell' ambito delle pièces prese in esame, si possono, a nostro avviso, stabilire dei rapporti tra i diversi personaggi. Come alla fine della tragedia diventa evidente il legame affettivo, inizialmente fondato sull'autorità, che unisce Sœur Angélique a Sœur Françoise: «Je demanderais à Dieu votre mort, plutôt que de vous voir signer» ed ancora «Je ne vous quitte pas: on ne quitte que ce qu'on cesse d'aimer» ${ }^{35}$, così Mère Marie de L'Incarnation e Blanche de la Force, nell'opera bernanosiana, sono unite in un rapporto da superiore a subalterna che si rivela, alla fine, di vera preoccupazione di Mère Marie per le sorti della giovane suora ${ }^{36}$. Allo stesso modo, Sœur Françoise e Blanche de la Force rivelano la purezza della loro anima nel sacrificio spontaneo della propria persona, mentre Sœur Angélique e Mère Marie ne dimostrano l'inadeguatezza l'una attraverso il dubbio e l'altra attraverso il peccato d'orgoglio, volendo il martirio ad ogni costo e confondendo, come vedremo, l'onore cristiano con l'onore del mondo. La pace ricercata e percepita da Sœur Françoise, il suo «unique nécessaire», e sottolineiamo quanto questo concetto sia montherlantiano ${ }^{37}$, è molto simile a quella di Sœur Constance nei Dialogues ed a quella di Chantal de Clergerie nella Joie. Queste tre figure d'enfant possono, infatti, dire: «Ce qu'il y a dans ma paix est l'unique nécessaire. [...] Je ne suis faite que pour l'adoration. Quand je ne suis pas devant Dieu, je suis toujours dans une sorte d'étonnement...»>38.

Parallelamente alle immagini della purezza anche i personaggi traditori sembrano essere speculari nell'opera montherlantiana ed in quella bernanosiana, in particolare in Port-Royal e nella Joie: il personaggio di Sœur Flavie, la delatrice che compila la lista delle dodici sorelle che saranno espulse dall'Arcivescovo, sembra riflettersi in Fiodor, l'autista russo che nella Joie, inizialmente attratto ed incuriosito dalla luce della santità di Chantal, arriva ad uccidere la fanciulla, sparandole in piena notte. Sia Sœur Flavie che Fiodor simboleggiano il tradimento di Giuda nel contesto della Passione di Cristo, ovvero nella persecuzione subita tanto dalle suore di Port-Royal quanto dai personaggi di Bernanos, in questo caso da Chantal de Clergerie.

Altro tema centrale che accomuna Port-Royal ai Dialogues des Carmélites e che si dimostra determinante nell'agire dei personaggi è il tema dell'onore. Essenzialmente sviluppato attraverso il personaggio di Mère Marie de l'Incarnation nei Dialogues, in Port-Royal il tema dell'onore coinvolge non solo Angélique Arnauld, la cui famiglia di origine è nota per la grande dignità e l'integrità morale, ma l'intero monastero.

Nei Dialogues, Bernanos mette in luce la distinzione tra l'onore cristiano e l'onore inteso secondo le regole del mondo. Per Bernanos, la vita e l'«honneur» delle suore sono riposti nelle mani di Dio e l'ordine del Carmelo non è di certo un ordine di cavalleria. Le religiose riunite in convento sono tutte uguali agli occhi di Dio, fra di loro non esiste

(34) G. Bernanos, Journal d'un curé de campagne, in Euvres romanesques, cit., pp. 1111-1113.

(35) H. De Montherlant, Port-Royal, in Théâtre, cit., pp. 912-914.

(36) Cfr. G. Bernanos, Dialogues des Carmélites, in Euvres romanesques, cit. pp. 1700-1703.

(37) Su questo tema rimandiamo al nostro studio Henry de Montherlant e la "quête du bonbeur", in «Studi Francesi», n. 144, settembre-dicembre 2004, pp. 487-511. Si veda in particolare p. 499. 
distinzione di classe, non esistono borghesi o aristocratiche, ciò che conta è la purezza dell'anima e la fedeltà al Padre. Anche la paura di Blanche e quindi il suo fragile carattere vanno considerati in questa prospettiva e cioè quali elementi naturali propri di una creatura di Dio: «LA PRIEURE: [...] Le Carmel n'est pas un ordre de chevalerie, que je sache! MÈre MARIE: [...] C'est le caractère qui manque à Blanche de la Force» ${ }^{39}$. Mère Marie de l'Incarnation è colei che fraintende il senso cristiano dell'onore e, come abbiamo già detto, si appella ad un concetto di «honneur» legato ai valori sociali e di classe.

In Port-Royal, il significato dell'onore cristiano è saldamente connesso all'interpretazione giansenista della religione. In questo contesto, Montherlant supera il cattolicesimo "all'italiana" conosciuto nella sua prima giovinezza e si avvicina con simpatia al «christianisme pris au sérieux» ${ }^{40}$. La scoperta di Port-Royal lo affascina per il suo alto contenuto di fede alla divinità di Gesù Cristo. Qui si instaura un altro punto di contatto col pensiero bernanosiano e cioè il grande senso di elevazione morale e spirituale che si traduce nell'avversione di entrambi gli autori per la mediocrità del sentire. L'esaltazione dell'anima, morale per Montherlant ed immortale per Bernanos, ha a che vedere con il senso di eccellenza e di perfezionamento spirituale dell'individuo. Nel giansenismo, già diffuso nella famiglia di Montherlant, Henry trova una corrispondenza di valori e di sentimenti che appartengono al côté più profondo ed incorruttibile del suo pensiero. Nei giansenisti, solitari rigorosi esclusi dalla grande società degli uomini mediocri, Montherlant riconosce dei fratelli spirituali e condivide la loro scelta del ritiro e dell'isolamento: «Dans le jansénisme je trouvais aussi des solitaires, des rigoureux, des dissidents, et une minorité: cette famille était et ne cessera jamais d'être la mienne» ${ }^{41}$. Raggiunta la consapevolezza della propria superiorità morale, la tentazione montherlantiana è quella del rifiuto del mondo e della retraite $e^{42}$. La lotta contro la mediocrità degli animi volgari è presente tanto nel pensiero montherlantiano quanto in quello bernanosiano. Port-Royal è il luogo per eccellenza in cui viene difesa la purezza del cristianesimo ed il Carmelo di Compiègne custodisce le anime elette delle suore che hanno fatto voto di martirio per dimostrare la loro fedeltà a Dio. Nota è anche la polemica bernanosiana, riscontrabile nell'opera saggistica e narrativa, contro la menzogna e la mediocrità dei "cattivi" preti che non sanno innalzare la propria anima verso la luce della grazia divina. La predilezione montherlantiana per la forza morale che contraddistingue le suore di Port-Royal deriva dalla sua condivisione delle dottrine stoiche. Egli porta l'esempio di Marc'Aurelio e ribadisce l'impegno delle anime eccezionali del monastero: «C'est la lutte entre ceux qui prennent tout à fait au sérieux, et ceux qui ne prennent pas tout à fait au sérieux, et la défaite inéluctable, toujours et en toute circonstance, des premiers ${ }^{43}$. Nel pensiero di Montherlant è insito anche il convincimento dell'ineluttabile sconfitta cui sono destinati coloro che perseguono un insegnamento di perfezionamento e di elevazione morale. Per Montherlant, che condivide il pensiero di Sainte-Beuve, i Francesi non sono stati in grado di imitare e di custodire l'alta virtù dei giansenisti ${ }^{44}$. Qui come altrove nell'opera saggistica e narrativa, basti pensare a La France et la morale de midinette, Henry rivolge l'accusa di fiacchezza ai suoi connazionali, incapaci di un innalzamento morale e spirituale di stampo stoico. Il senso di appartenenza ad una minoranza di anime elette è presente in Montherlant già nel saggio Les Chevaleries, contenuto nel Solstice de Juin. Nelle Chevaleries, Henry enuncia il suo modello

(38) H. De Montherlant, Port-Royal, in Théatre, cit., pp. 871-872.

(39) G. Bernanos, Dialogues des Carmélites, in Euvres romanesques, cit., pp. 1616-1617.

(40) Cfr. H. DE MONTHERLANT, Sur «Port-Royal», in Théâtre, cit., p. 526.

(41) Ibid.
(42) Cfr. H. DE Montherlant, Port-Royal et la "Grande Tentation", in Théâtre, cit., pp. 924-926.

(43) H. DE MONTHERLANT, Du côté de la souffran$c e$, in Théâtre, cit., pp. 917-918.

(44) Cfr. H. DE Montherlant, Port-Royal et le puritanisme romain, in Théâtre, cit., p. 923. 
cavalleresco, costituito nel 1919 alla fine della prima guerra mondiale, sottolineando il carattere di superiorità morale degli appartenenti al circolo degli eletti e mescolando a quello stesso modello il rigore del giansenismo. Per Montherlant, lo spirito della riforma della cristianità, quello espresso in Port-Royal, deve animare anche lo spirito della riforma della nazione di cui egli sente l'esigenza nel Solstice de Juin ${ }^{45}$. I valori del cristianesimo e del giansenismo sono rintracciabili in molte opere di Montherlant, ad esempio in Don Fadrique, Service Inutile, Les jeunes filles. In questo contesto, PortRoyal rappresenta la pièce in cui le virtù cristiane sono grandemente elogiate insieme con la forza morale di matrice stoica. Tale necessità di ascesi spirituale e morale è comune, come abbiamo constatato, ai Dialogues ed all'opera bernanosiana in generale.

Benché Port-Royal sia incentrato sull'esaltazione delle virtù gianseniste, all'interno della pièce Montherlant si sofferma sia sul côté giansenista sia su quello cattolico ${ }^{46}$. Il sincretismo religioso dell'autore, rintracciabile per esempio in Malatesta dove il paganesimo si mescola al cattolicesimo, è presente anche in Port-Royal ${ }^{47}$. Tuttavia, nonostante la coesistenza della dottrina cattolica e di quella giansenista, nella pièce montherlantiana si coglie la predilezione dell'autore per il rigore giansenista e per la continua ricerca dell'ascesi spirituale dell'individuo.

L'individualismo di Montherlant, diffuso in tutta l'opera ed anche in Port-Royal, è facilmente accostabile all'individualismo bernanosiano dei Dialogues des Carmélites: in entrambe le rappresentazioni teatrali all'individuo è conferita grande dignità e capacità di elevazione morale sino ai livelli più sublimi dell'innalzamento spirituale. Sia Montherlant che Bernanos sono, inoltre, contro l'omogeinizzazione della civiltà di massa ed a favore della salvaguardia dei valori monarchici ed aristocratici. Entrambi riconoscono, tuttavia, che la vera nobiltà è quella morale e che il primato dei valori va conferito al rispetto per la vita e per la libertà individuale. Se per Montherlant la libertà si configura come la ricerca dell'«unique nécessaire», il diritto alla retraite ed alla feinte, cioè la consapevolezza della vanità delle regole del contesto sociale, si pensi a Le Songe ed a Service Inutile, per Bernanos la libertà dell'essere umano coincide con la forza della fedeltà spirituale e con l'affrancamento da ogni sopruso ed ingiustizia sociale. Nei Dialogues come in Port-Royal, l'individuo consolida la propria libertà acquisendo la consapevolezza del Nulla che circonda la profonda realtà spirituale del convento e del monastero. La rinuncia del mondo e il distacco nei confronti di tutto ciò che rappresenta la vana ambizione degli uomini sono lo strumento di libertà delle anime eccezionali di Compiègne e di Port-Royal.

Accomunate dal tema della paura che acquisisce nei differenti contesti significati che rimandano alla nozione di verità cristiana per Bernanos e di dominio stoico per Montherlant ${ }^{48}$, i Dialogues des Carmélites e Port-Royal tramandano un messaggio universale e atemporale riguardante la contestazione della persecuzione nei confronti delle minoranze sociali. La sofferenza e la paura delle suore perseguitate si rivelano attuali e paragonabili, secondo Montherlant che non dimentica di citare Bernanos ed i suoi Dialogues", a quelle degli europei contemporanei di Henry: «L'opinion serait ancore plus de ce "côté de la souffrance", si elle réalisait combien la persécution fut

(45) Cfr. H. DE Montherlant, Sur «Port-Royal», in Théâtre, cit., p. 528.

(46) Ibid., p. 529.

(47) Cfr. H. DE Montherlant, Sur le bord de l'océan religieux, in Théâtre, cit., p. 948.

(48) Cfr. H. De Montherlant, Du côté de la souffrance e Notes sur «Port-Royal», in Théâtre, cit., pp. 919 e 945.

(49) «C'est longtemps aussi après avoir choisi et conçu le sujet du second Port-Royal que je me suis souvenu que, dans l'œuvre de théâtre la plus récente consacrée à une communauté féminine - les Dialogues des Carmélites, - le sentiment dominant était le même qu'ici: la peur. La peur, ou deux peurs différentes, mais qui toutes deux ont leur source aux humbles entrailles de la créature humaine. Les moralistes de l'avenir pourront se demander si, par cela, ces œuvres ne sont pas bien de notre époque» (Du côté de la souffrance, cit., p. 919). 
alors semblable à celles d'aujourd'hui; à quel point les jansénistes sont des frères et des sœurs pour les Européens d'aujourd'hui» ${ }^{50}$.

Partendo da presupposti ideologicamente lontani, almeno in apparenza, Bernanos e Montherlant si rivelano, al contrario, quanto mai vicini per la loro costante ricerca del senso sublime dell'esistenza umana, per l'uno rintracciabile nell'incontestabile verità della fede, per l'altro nella conquista intima e personale di un assoluto terreno.

PIERANGELA ADINOLFI

(50) Ibid. Sui temi dell'opera bernanosiana proposta si vedano: G. GAUCHER, Le thème de la mort dans les romans de Bernanos, avec des inédits présentés par A. BÉGUIN, Paris, Lettres Modernes, 1955, pp. 88-93; J. L. GILLESPIE, Le tragique dans l'cuvre de Georges Bernanos, Genève, Droz, Paris, Minard, 1960; W. BusH, Souffrance et expiation dans la pensée de Bernanos, Paris, Minard - Lettres Modernes, 1962; Y. BRIDEL, L'esprit d'enfance dans l'auvre romanesque de Georges Bernanos, Paris, Minard - Lettres Modernes, 1966; D.P. MACCHI, Bernanos e il problema del male, Varese, La Lucciola, 1958; M. Estève, Le sens de l'amour dans les romans de Bernanos, Paris, Minard, 1959 e Bernanos, Paris, Gallimard, 1965; H. URs VON Balthasar, Le Chrétien Bernanos, traduit par M. Gandillac, Paris, Seuil, 1956; G. Gaucher, Georges Bernanos ou l'invincible espérance, Paris, Plon, 1962; C. Bo, La réalité de Bernanos, in «Etudes bernanosiennes», Témoin de l'bomme, témoin de Dieu, 3-4, 1963, pp. 7-26; M. Molinari, Donissan, le champion de Dieu, in «Etudes bernanosiennes», Témoin de l'bomme, témoin de Dieu, 3-4, 1963 , pp. 109-137; J. Pfeifer, La Passion du Christ et la structure de «Dialogues des Carmélites», in «Etudes bernanosiennes», Témoin de l'bomme, témoin de Dieu, 3-4, 1963, pp. 141-169; P. RENARD, Georges
Bernanos, traduzione di G. BARGIONI, Milano, Mursia, 1970, Le gouffre de lumière, in «Revue

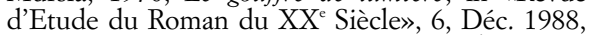
pp. 71-79, Georges Bernanos, témoin, Toulouse, PU du Mirail, 1994 e Figures esthétiques de l'exigence éthique dans quelques textes des littératures romanes contemporaines, in «Lettres Romanes», 2000, pp. 93-104; PH. Le Touze, Bernanos et l'expérience de Dieu, in «Recherches et Travaux», 58, 2000, pp. 141-47 ; E. BENOIT, De la crise du sens à la quête du sens (Mallarmé, Bernanos, Jabes), Paris, Cerf, 2001; C. BARTHE, Bernanos, la peur ou l'insondable de Dieu, in «Travaux de Littérature», 16, 2003, pp. 105-121 ; D. Perrot-Corpet, Écrire devant l'absolu: Georges Bernanos et Miguel de Unamuno, Paris, Champion, 2005. In merito ai temi montherlantiani trattati si vedano inoltre: J. DE BEER, Montherlant ou l'bomme encombré de Dieu, Paris, Flammarion, 1963; PH. DE SAInT Robert, Montherlant le séparé, Paris, Flammarion, 1969 e Montherlant ou la relève du soir, Paris, Les Belles Lettres, 1992; P. D’ARX, La ferme dans le théatre de Henry de Montherlant, Paris, Nizet, 1973; P. SIPRIOT, Montherlant sans masque, Paris, Laffont, 1990; S. Hillen, Le Roman monologue. Montherlant, auteur, narrateur, acteur, Paris-Caen, Lettres Modernes Minard, 2002. 